\title{
Dual-Band Full-Duplex Tx/Rx Antennas for Vehicular Communications
}

\begin{abstract}
This paper proposes a novel, highly integrated and full-duplex antenna/array for intelligent transport systems (ITS) applications. Different from traditional single-port antennas, this genre antenna has two highly isolated ports with different operation bands (channels). It supports the full-duplex operation mode fulfilling uplink and downlink simultaneously. The other contribution of this work is that the antenna combines several functions, such as filtering, duplexing and radiating, into one single component, forming a multifunctional device. This highintegration design approach leads to the removal of the traditionally separated filters, duplexers and the interfaces between them, resulting in a compact $R F$ frontend with reduced size, weight and complexity. These properties could also reduce the potential cost of the ITS. Low-cost microstrip antenna is employed in this design and the two operation bands are achieved by appropriately arranging the locations and couplings among the resonators. To improve the channel isolation and suppress out-of-band interference, cross-coupling is introduced to generate transmission nulls. To verify the concept, one antenna element and two $2 \times 2$ arrays at $C$-band are designed, prototyped and tested. All the measurements agree well with the simulations, showing two full-duplex channels of $4.58-4.83 \mathrm{GHz}$ and 5.86 $6.2 \mathrm{GHz}$ for transmitting and receiving, respectively. The proposed antennas also show excellent performance in terms of channel isolations, frequency selectivity, out-of-band rejections, radiation patterns and gains.
\end{abstract}

Index Terms - Antenna, antenna array, duplexing, filtering, highly integrated, low cost, vehicular communications.

\section{INTRODUCTION}

TTELLIGENT Transportation Systems (ITS) will significantly Iinfluence our future daily life as they emerge to provide numerous applications related to traffic safety and traffic efficiency. Vehicular communication plays an important role in the realization of these applications as it enables the direct information exchange between vehicles. In the past decade, lots of effort have been put into the research on the applications, architectures, protocols and channel models of vehicular communications [1]-[12]. However, very little work focusing on the radio frequency (RF) frontends of the vehicular communication has been reported in the open

Manuscript submitted on February 27, 2017; This work is supported by UK EPSRC grant EP/N032497/1 and YW is supported by UK EPSRC under Contract EP/M013529/1

C. X. Mao and S. Gao are with School of Engineering and Digital Arts, University of Kent, UK (cm688@kent.ac.uk).

Y. Wang is with the Department of Engineering Science, University of Greenwich, UK.

literature.

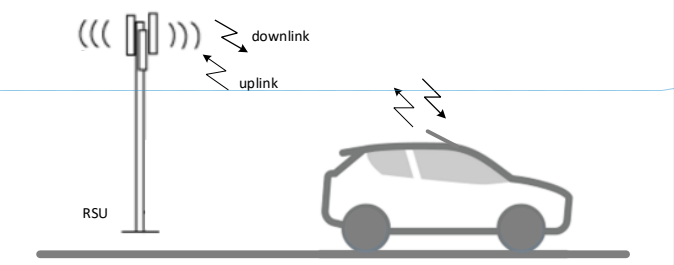

(a)
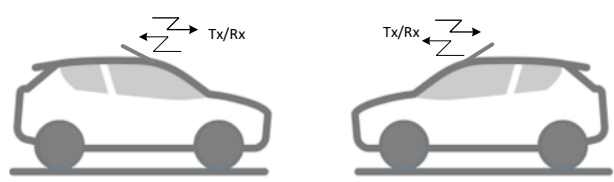

(b)

Fig. 1. Two vehicular communication scenarios: (a) vehicle-to-infrastructure communication, (b) vehicle-to-vehicle communication.

Since the RF frontends will support the transmission and reception of the signals between vehicles and the requirements of the vehicular-based communications are usually different from the traditional wireless communications, it is worthwhile to pay more attention to the research on the vehicular-based RF frontends [13].

The vehicular communication can be classified into two application scenarios: vehicle-to-infrastructure communication and vehicle-to-vehicle communication, as shown in Fig. 1. For the vehicle-to-infrastructure communication, as shown in Fig. 1(a), the transceiver on-board exchanges information with a roadside unit (RSU), which is a part of the infrastructure. The applications of this kind include: intersection collision warning, emergency vehicle warning, wrong way driving warning, traffic condition warning and signal violation warning, etc [2]. In ITS, information can also be exchanged or shared between vehicles, as shown in Fig. 1(b). This real-time communication can effectively reduce the driving risk by providing the warnings such as intersection collision, overtaking collision, head on collision and rear end collision. Both scenarios require the RF frontends of the communication entities to facilitate the full-duplex operation where the transmission and reception can be processed simultaneously. The RF frontends (the antennas in particular) are also demanded to have high isolation between the $\mathrm{Tx}$ and
Commented [YW1]:

Commented [YW2]: ? 


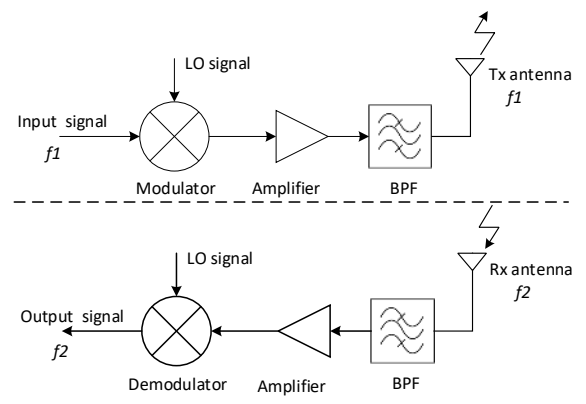

(a)

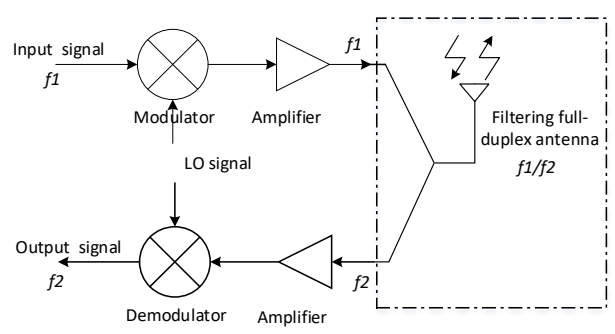

(b)

Fig. 2. The block diagrams of (a) a traditional separated Rx/Tx system, (b) an integrated Rx/Tx system.

Rx channels, high frequency selectivity and good out-of-banc rejection performance so as to avoid the potential interferences. Moreover, the economical factor should be taken into consideration as the vehicular communication systems would be massively produced. This paper focuses on the RF frontend design for the vehicle-to-infrastructure scenario.

To meet these requirements, the integrated design of multifunctional RF frontends is an attractive solution. During the past several years, a lot of works that aim at integrating the passive components such as filters, power dividers, duplexers and antennas into one single device have been reported [14] [20]. Such integrated designs can effectively reduce the complexity of the RF frontends while providing an improved frequency response. However, these works did not fully consider the requirements of full-duplex operation and compactness for the future vehicular communications. To address this research gap, this paper proposes a novel highlyintegrated full-duplex $\mathrm{Tx} / \mathrm{Rx}$ antenna with improved frequency selectivity, channel isolation and out-of-band rejection. In addition, two antenna arrays are conceived to enhance the gain.

\section{RF FRONTEND ARCHITECTURES AND SPECIFICATIONS}

A. RF frontend architecture

To support the full-duplex $\mathrm{Rx} / \mathrm{Tx}$ operations in vehicular communications while mitigating the interferences between the

TABLE-I

SPECIFICATIONS OF THE FULL-DUPLEX ANTENNA

\begin{tabular}{cc}
\hline \hline Frequency bands & Uplink: $f l=4.7 \mathrm{GHz} ;$ \\
Downlink: $f 2=6 \mathrm{GHz}$ \\
Fractional bandwidths & Low-band: $5.0 \%$; high-band: $5.0 \%$ \\
Polarization characteristics & Vertical polarization \\
Isolation between bands & $30 \mathrm{~dB}$ \\
Cross-polarization level & $-30 \mathrm{~dB}$ \\
Gain fluctuation in-bands & $\pm 0.5 \mathrm{~dB}$ \\
Inter-channel gain suppression & $20 \mathrm{~dB}$ \\
Antenna element size & $0.5 \lambda \times 0.5 \lambda$ \\
\hline \hline
\end{tabular}

Rx/Tx channels, two different frequency bands are usually used in receiving and transmitting modules. Fig. 2 compares the block diagrams of a conventional separated $\mathrm{Rx} / \mathrm{Tx}$ systems and the proposed integrated $\mathrm{Rx} / \mathrm{Tx}$ system. For the conventional system in Fig. 2(a) the Rx and Tx system have two independent processing blocks with different frequencies. One of the disadvantages is that two groups of antennas and bandpass filters are required to facilitate the full-duplex operation, which leads to a bulky and complicated RF frontend at both vehicle side and infrastructure side.

Looking at the block diagram in Fig. 2(b), the Tx antenna and the Rx antenna are replaced by one single dual-port fullduplex antenna, which operates at $f 1$ and $f 2$ simultaneously while providing a high channel isolation. Furthermore, the separate bandpass filters are removed by the integrated filtering characteristics of the full-duplex antenna. The size, weight, complexity and therefore the cost of the system can be significantly reduced. Such a compact full-duplex antenna is most suitable for the applications of vehicular communication.

\section{B. Specifications}

The targeted specifications for the full duplex filtering antenna are listed in Table-I. The antenna works at C-band and the central frequencies for the uplink and downlink operations are 4.7 and $6.0 \mathrm{GHz}$, respectively. The fractional bandwidths (FBW) for both bands are required to be above 5\%. The two operation bands are expected to have consistent vertical polarization characteristics. An inter-channel isolation of 30 $\mathrm{dB}$ between the uplink and downlink is demanded. The antenna is also required to have a high polarization purity with the cross-polarization level lower than $-30 \mathrm{~dB}$ at both operations. Additionally, a flat gain response $( \pm 0.5 \mathrm{~dB})$ in the passbands and high inter-channel gain suppression $(20 \mathrm{~dB})$ are targeted. To further conceive an array antenna, the duplex antenna element, including the two feeding/filtering channels, should be confined in an area of $0.5 \lambda \times 0.5 \lambda$. These stringent specifications lead to a number of challenges in the design.
Commented [YW3]: This comparison may be an overstatement. As far as I know, most of the full-duplex system is like Fig. 2(b). As far as I know, most
Fig. 2(a) is rarely used.

Fig. 2(a) is rarely used. Probbaly you should focus on the comp duplexer-antenna and an integrated one. 


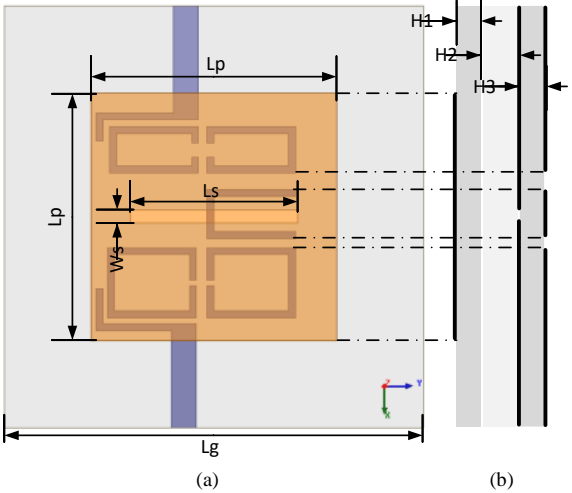

(b)

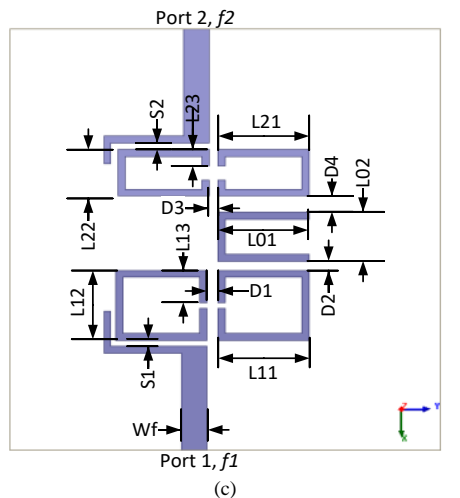

(c)

Fig. 3. Configuration of the proposed dual-port full-duplex antenna element: (a) front view, (b) side view and (c) back view.

\section{DUAL-PORT Full-DuPlex ANTENNA ElEMENT}

In this section, the structure of the dual-port full-duplex antenna element and its operation mechanism are first explained by using the coupling topology and the corresponding coupling matrix. Then, the approach of realizing the full-duplex is illustrated. To further improve the frequency selectivity and out-of-band rejection, the implementation of non-adjacent cross-coupling is discussed.

\section{A. Configuration}

Fig. 3 shows the configuration of the proposed dual-port full-duplex filtering antenna element. It has a stacked structure, containing two substrates and a thin Rohacell foam between them. Viewing from the front, the radiation element is a square patch printed on the top layer of the upper substrate, as shown in Fig. 3(a). The feeding networks and the resonators of the two channels are placed on the bottom layer of the lower substrate, as shown in Fig. 3(c). There are two sets of open-loop resonators with different resonant
TABLE-II

PARAMETERS OF THE PROPOSED ANTENNA: (MM)

\begin{tabular}{ccccccccc}
\hline \hline $\mathrm{Lp}$ & $\mathrm{Lg}$ & $\mathrm{Ls}$ & $\mathrm{Ws}$ & $\mathrm{H}_{1}$ & $\mathrm{H}_{2}$ & $\mathrm{H}_{3}$ & $\mathrm{Wf}$ & $\mathrm{L}_{01}$ \\
17.6 & 50 & 12 & 0.9 & 0.813 & 1 & 0.813 & 1.8 & 6.35 \\
\hline $\mathrm{L}_{02}$ & $\mathrm{~L}_{11}$ & $\mathrm{~L}_{12}$ & $\mathrm{~L}_{13}$ & $\mathrm{~L}_{21}$ & $\mathrm{~L}_{22}$ & $\mathrm{~L}_{23}$ & $\mathrm{~S}_{1}$ & $\mathrm{~S}_{2}$ \\
3.5 & 6.35 & 5 & 2.3 & 6.35 & 3.3 & 1.15 & 0.4 & 0.4 \\
\hline $\mathrm{D}_{1}$ & $\mathrm{D}_{2}$ & $\mathrm{D}_{3}$ & $\mathrm{D}_{4}$ & & & & & \\
0.75 & 0.65 & 1.15 & 0.6 & & & & & \\
\hline
\end{tabular}

frequencies $\left(f_{1}\right.$ and $\left.f_{2}\right)$ adopted as the channel filters for the uplink and downlink operations. They are joined together by a hairpin resonator in the middle. It should be noted that the hairpin and the patch are synchronically tuned to $f_{0}$ of 5.4 $\mathrm{GHz}$, which is different from the $f_{1}$ and $f_{2}$ of the two filtering channels. The patch and the feeding structures/resonators share the same ground plane, which is printed on the top layer of the bottom substrate. Such configuration not only makes the design more integrated but also reduce the adverse interference between the radiation unit and the feeding structures/resonators. A slot-line etched in the ground plane is used to enable the electromagnetic coupling between the hairpin resonator and the patch.

In this work, half wavelength resonators (open-loop and hairpin) are used. The relationship between the lengths of the resonators and the resonant frequencies can be approximately calculated using the following expressions [21],

$$
\begin{aligned}
& 2 \cdot\left(L_{11}+L_{13}\right)+L_{12} \approx \frac{c}{2 \cdot f_{1} \sqrt{\varepsilon_{r}}} \\
& 2 \cdot\left(L_{21}+L_{23}\right)+L_{22} \approx \frac{c}{2 \cdot f_{2} \sqrt{\varepsilon_{r}}} \\
& 2 \cdot L_{01}+L_{02} \approx \frac{c}{2 \cdot f_{0} \sqrt{\varepsilon_{r}}} \approx L_{p}
\end{aligned}
$$

where $c$ is the speed of the light in free space, $\varepsilon_{r}$ is the effective permittivity. $f_{1}=4.7 \mathrm{GHz}$ and $f_{2}=6 \mathrm{GHz}$ are the central frequencies of the two operations, respectively. $f_{0}=5.4 \mathrm{GHz}$ is the resonant frequency of the hairpin and the patch. Simulations and optimizations were performed using High Frequency Structural Simulator (HFSS) and the optimized dimensions are presented in Table-II.

\section{B. Topology and coupling matrix}

The full-duplex antenna in Fig. 3 can be represented using a coupled-resonator network, as shown in Fig. 4. The circles represent single-mode resonators whereas the lines represent the electromagnetic couplings between them. It should be noted that the radiating patch (Resonator-1) also serves as a single-mode resonator. The proposed duplexing antenna is essentially an all-resonator based duplexer with its common port a radiator. The hairpin, as the junction resonator, joins the two sets of resonators as the channel filters. It replaces the traditional transmission-line based junctions in duplexers. The patch and the hairpin both resonate at around $f_{0}$. The coupling 
$m_{12}$ between them is designed to produce two separate resonant modes $\left(f_{1}\right.$ and $\left.f_{2}\right)$ corresponding to the two channel frequencies. These resonances

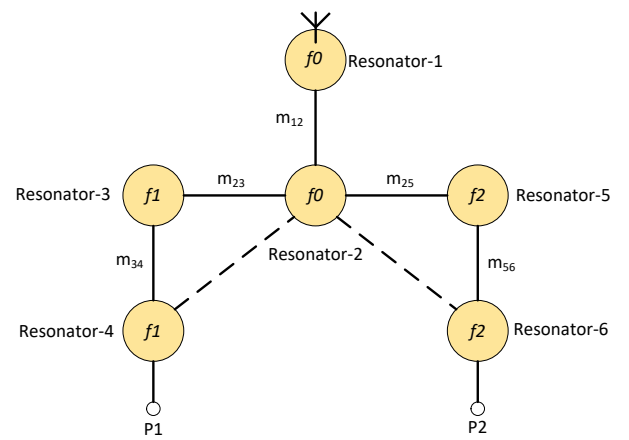

Fig. 4. The topology of the proposed full-duplex antenna in the form of coupled resonators.

are coupled to the two filtering channels via $m_{23}$ and $m_{25}$. A six-resonator (or six-pole) duplexer is formed (including the one-pole from the patch radiator) and each channel is designed to have a $3^{\text {rd }}$-order filtering characteristic. It should be noted that cross-couplings between non-adjacent resonators are deliberately introduced between the resonator- 2 and the resonator-4 (resonator-6), as indicated by the dash line in Fig. 4. These are used to produce transmission zeros for improving the isolation between the two channels and out-of-band rejection, which will be discussed later. In the topology of Fig. 4 , three types of resonators with different resonant frequencies $\left(f_{0}, f_{1}\right.$ and $f_{2}$ ) are integrated together, leading to a very compact device.

The abovementioned couplings $m_{i j}$ can be contained in a single coupling matrix $[m]$ that describes the three-port network in Fig. 4. This matrix is linked to the better-known Sparameters of the network via a normalized matrix [A] [22].

$$
[A]=[q]+p[U]-j[m]
$$

where $[U]$ is an identity matrix, $[m]$ is the normalized coupling matrix. $[q]$ is a diagonal matrix with only three non-zero elements $[q]_{i i}=1 / q_{e, i}(i=1,4,6)$ in this case, where $q_{e, i}$ is the normalised external quality factor from the port attached to the $i$-th resonator. In the case of Fig. 4, the 'port' attached to resonator-1 (the patch) is equivalent to the free space. So, $q_{e, 1}$ is essentially the coupling from the patch to the free space The scattering parameters of the network can be expressed as

$$
S_{11}= \pm\left(1-\frac{2}{q_{e, 4}}[A]_{44}^{-1}\right), \quad S_{22}= \pm\left(1-\frac{2}{q_{e, 6}}[A]_{66}^{-1}\right)
$$

The transmission coefficients can be expressed similarly as in [22]. But these are less relevant in this case as the antenna 'port' is not physically measurable. In the real frequency domain, the external quality factors and the coupling matrix can be found by $Q_{e, i}=q_{e, i} / F B W$ and $M_{i j}=m_{i j} \times F B W$. To guide the initial design in this work, the coupling matrix $M$ is synthesized for the topology in Fig. 4. For simplicity, the weak cross couplings are ignored at this stage. The optimization based synthesis method is used to inversely obtain $M$ from the expected S-parameter responses (e.g. bandwidth and return losses) [22]. For the $5 \%$ low-band centered at $f_{l}=4.7 \mathrm{GHz}, 5$ $\%$ high-band centered at $6 \mathrm{GHz}$ and the return loss of $10 \mathrm{~dB}$, the following $6 \times 6$ coupling matrix $M$ is found,

$$
M=\left[\begin{array}{cccccc}
0 & 0.2546 & 0 & 0 & 0 & 0 \\
0.2546 & 0 & 0.0586 & 0 & 0.0603 & 0 \\
0 & 0.0586 & -0.2377 & 0.0405 & 0 & 0 \\
0 & 0 & 0.0405 & -0.2466 & 0 & 0 \\
0 & 0.0603 & 0 & 0 & 0.2356 & 0.0411 \\
0 & 0 & 0 & 0 & 0.0411 & 0.2476
\end{array}\right]
$$

where $M_{i j}$ represents the de-normalized coupling coefficient between the resonator $i$ and resonator $j$. The external quality factors that represent the coupling between the port P1 and Resonator-4 and between the port P2 and Resonator-6 are $Q_{e, 4}$ $=33.4$ and $Q_{e, 6}=34.4$. From the patch to the free space, this is $Q_{e, 1}=8.7$. These are all obtained together with the $M$ matrix.

\section{Resonator-coupled dual-band patch antenna}

As can be observed in the coupling matrix, strong coupling $\left(M_{12}=0.2546\right)$ exists between the patch and the hairpin resonator. This strong coupling helps to achieve the dual-band impedance matching at the two designated operation bands while maintaining a consistent radiation polarization. Fig. 5 illustrates the configuration of the proposed resonator-coupled patch antenna. Using full-wave simulation, the ratio of the two bands $\left(f_{2} / f_{l}\right)$ as the function of the length of the slot $(L s)$ is obtained and presented in Fig. 6. By adjusting $L s$ and therefore the coupling strength between 


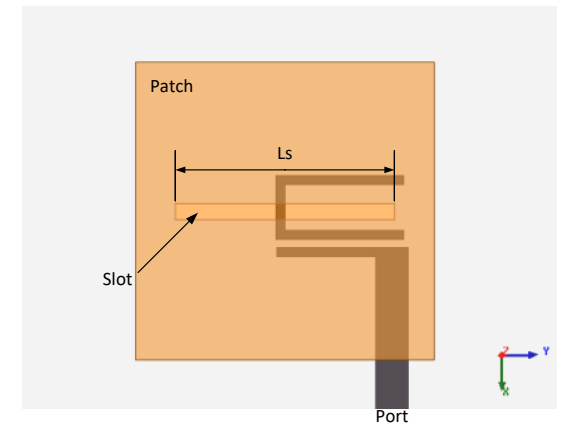

Fig. 5. Configuration of the dual-band hairpin-patch antenna.

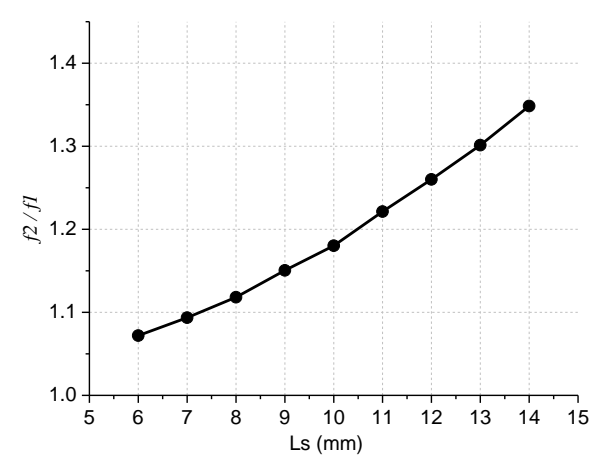

Fig. 6. The frequency ratio $\left(f_{2} / f_{1}\right)$ of the dual-band antenna in Fig. 5 as a function of the length of the slot, $L s$.

the patch and the resonator, the frequency ratio $\left(f_{2} / f_{1}\right)$ of the dual-band patch antenna can be tuned. When $L s$ is $6 \mathrm{~mm}$, the two resonant frequencies are very close to each other $\left(f_{2} / f_{1}=\right.$ 1.07). The frequency ratio increases to more than 1.35 as $L s$ increases to $14 \mathrm{~mm}$. It should be noted that the width of the slot has a similar effect on the two resonant frequencies, which is not presented here for brevity. To achieve the two frequency bands of 4.7 and $6.0 \mathrm{GHz}$ in this design (frequency ratio is 1.28), $L s$ is found to be $12 \mathrm{~mm}$.

\section{Cross-coupling}

Usually, the isolation between the two duplexing channels can be enhanced by using higher order filters, but this will increase the volume and insertion loss of the device. Here, the $3^{\text {rd }}$-order filtering channels are adopted. To further improve the isolation and out-of-band rejection of the duplexing filtering antenna while maintaining a compact size, cross couplings between non-adjacent resonators are introduced to generate additional transmission zeros. To illustrate this concept, two configurations of $3^{\text {rd }}$-order bandpass filters with different layouts are compared. As shown in Fig. 7(a), the first one is a cascaded layout, denoted Type-I. Fig. 7(b) shows the layout adopted in this work (Type-II), where cross coupling is introduced between resonator 1 and 3 .

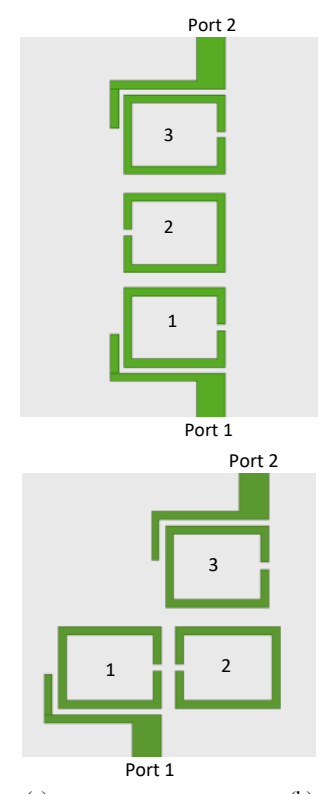

(a)

(b)

Fig. 7. Two configurations of $3^{\text {rd }}$-order band-pass filters with different layouts: (a) cascaded, Type-I, (b) adopted in this design, Type-II.

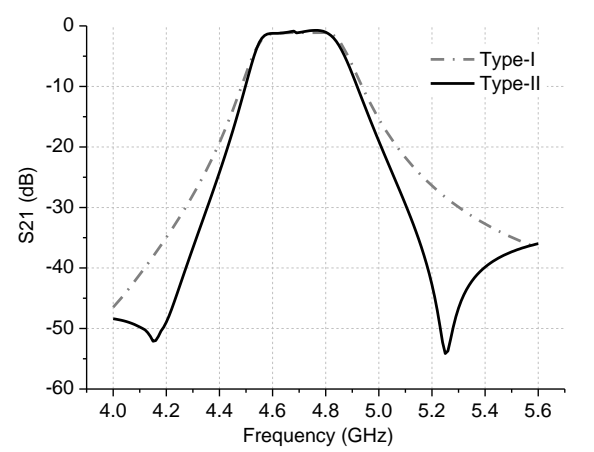

Fig. 8. Comparison of transmission responses between the two bandpass filters in Fig. 7.

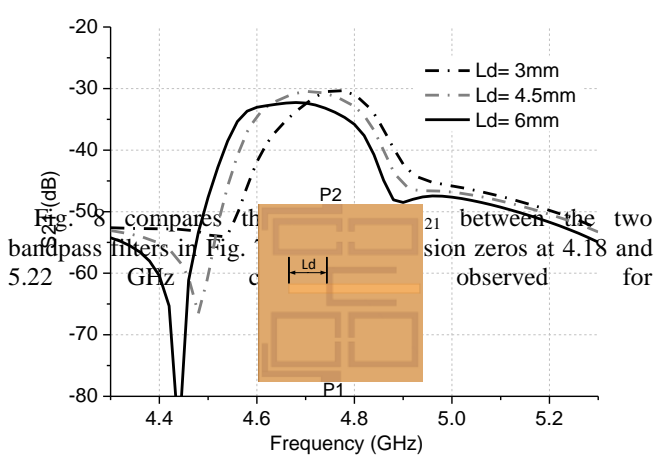




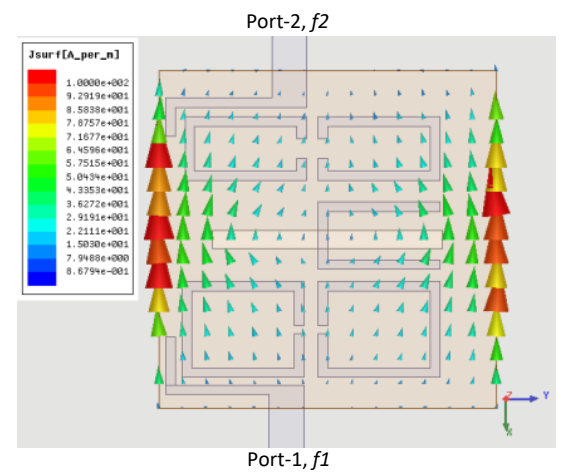

(a)

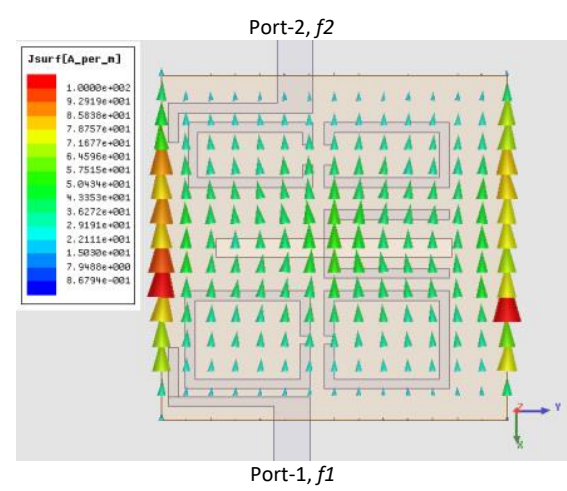

(b)

Fig. 10. Simulated current distribution: (a) Port 1 excitation, $4.7 \mathrm{GHz}$, (b) Port 2 excitation, $6 \mathrm{GHz}$

the Type-II filter, which contributes to the improved frequency selectivity and out-of-band rejection. Compared with the Type-I filter, the attenuation at $4.2 \mathrm{GHz}(5.2 \mathrm{GHz})$ is increased from $35 \mathrm{~dB}(26 \mathrm{~dB})$ to $50 \mathrm{~dB}(42 \mathrm{~dB})$. When the cross coupling configuration is used in the duplexing antenna design, the isolation between the two channels can be improved without increasing its footprint. Fig. 9 shows the $S_{2}$ (isolation) between the two channels/ports as a function of the locations of the hairpin resonator, measured by $L d$ in the inse of Fig. 9. This location is used to tune the cross coupling. It can be seen that the transmission zeros move to lower frequency as $L d$ increases.

\section{E. Current distribution}

Fig. 10 shows the simulated current distribution on the patch of the proposed dual-port full-duplex antenna. When Port 1 is excited at $4.7 \mathrm{GHz}$, as shown in Fig. 10(a), the current on the patch is symmetric but its path is slightly distorted around the slot line. The extended current path leads to an operation frequency lower than the patch's intrinsic resonance. When Port 2 is excited at $6 \mathrm{GHz}$, as shown in Fig. $10(b)$
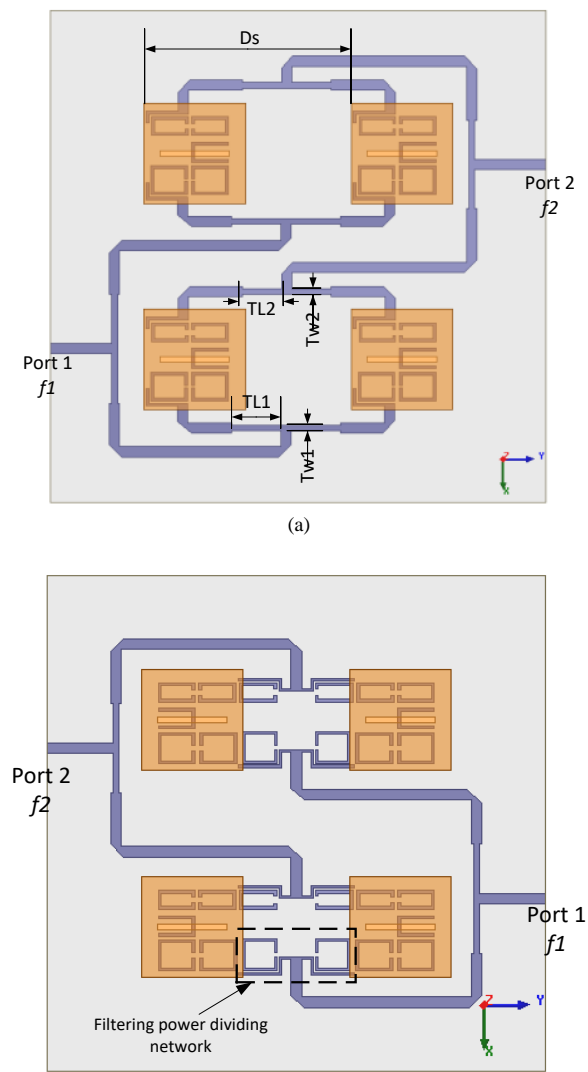

(b)

Fig. 11. Configurations of the two $2 \times 2$ full-duplex antenna arrays: (a) Array-I, with a $3^{\text {rd }}$-oder filtering response and a traditional quarterwavelength transformer feeding network; (b) Array-II, with a $4^{\text {th }}$-order filtering response and a novel filtering power dividing network. $D s=36$ $\mathrm{mm}, T L 1=8.6 \mathrm{~mm}, T L 2=6.8 \mathrm{~mm}, T w 1=1 \mathrm{~mm}, T w 2=1 \mathrm{~mm}$.

similar current distribution but with a shorter path can be seen. It is evident that the antenna works at its fundamental mode (TM01) for both frequencies (channels). As a result, the fullduplex antenna has the consistent radiation and polarization characteristics at the two operation bands.

\section{Full-DuPleX ANTENNA ARRAY}

To increase the channel capacities of the $\mathrm{Tx} / \mathrm{Rx}$ devices, especially for the RSUs, array antennas are always desired in the applications. Thanks to the compact size of the proposed full-duplex element in Fig. 3 (less than $0.5 \lambda$ ), it is very suitable to expand to an array. In this section, two full-duplex $2 \times 2$ arrays with different feeds and different orders of 
filtering are presented.

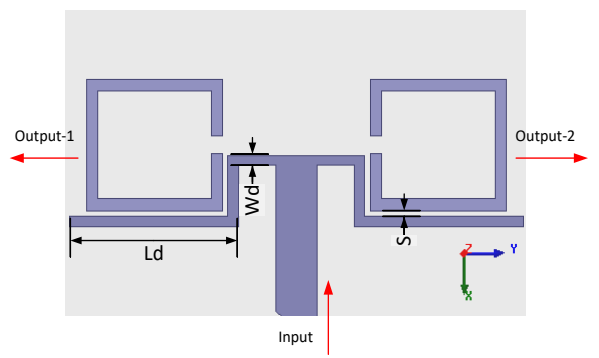

Fig. 12. Configuration of the 2-way filtering power dividing network in Array-II. $L d=6.8 \mathrm{~mm}, W d=0.45 \mathrm{~mm}, S=0.35 \mathrm{~mm}$.

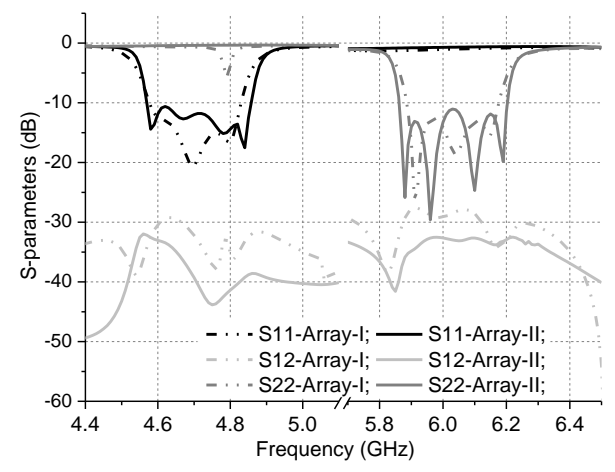

Fig. 13. Comparison of simulated S-parameters between the two array antennas.

\section{A. Array-I}

Fig. 11(a) shows the configuration and feeding networks of the first dual-port full-duplex antenna array, donated as ArrayI. It is based on the antenna element presented in Fig. 3 Because the two channels share the same aperture (patch), the spacing between the radiation elements is a trade-off between the efficiency and side-lobe level at the two operation bands. The spacing is chosen to be $D s=36 \mathrm{~mm}(? \lambda \square \square \square ? \square \square \mathrm{Hz})$.

The feeding networks for the two operation bands are designed separately. Port 1 is assigned to feed the low-band operation, whereas Port 2 is used for the high-band. The conventional 4-way power dividers with quarter wavelength transformers are used. The width of the $50 \Omega$ feed lines is 1.8 $\mathrm{mm}$. For the low-band operation, the length and width of the transformer are $T L l=8.6 \mathrm{~mm}$ and $T w l=1 \mathrm{~mm}$, respectively. For the high-band operation, the length and width are $T L 2=$ $6.8 \mathrm{~mm}$ and $T w 2=1 \mathrm{~mm}$.

\section{B. Array-II}

To further increase the order of the channels and therefore the frequency selectivity of the full-duplex array, another array is proposed based on a filtering power-dividing network, as

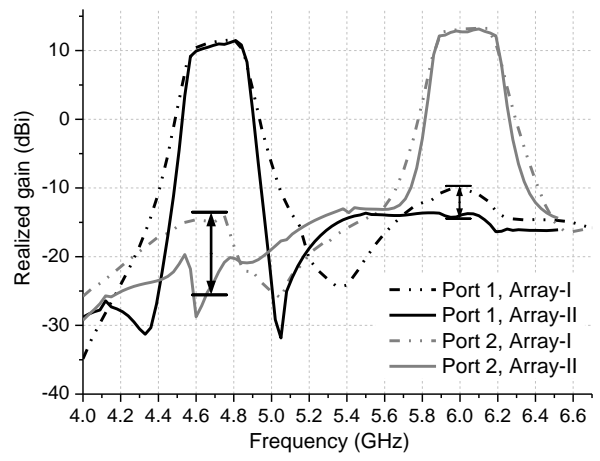

Fig. 14. Comparison of the simulated gains between Array-I and Array-II.

shown in Fig. 11(b). Different from the Array-I, the quarter wavelength transformers are replaced with a resonator-based power dividing network. An enlarged view of it is shown in Fig. 12. The input power is coupled directly from the feed line to two open loop resonators of $f 1$ and $f 2$, respectively. Each of these resonators is then coupled to a channel of the duplexing antenna element without using any impedance transformers. Such an integrated design increases the orders of the filtering channels from 3 to 4 without increasing its size. As a result, the frequency responses such as selectivity, bandwidths, isolations and out-of-band rejection can be improved.

\section{Filtering and harmonics suppression}

Fig. 13 compares the simulated S-parameters of the two proposed array antennas. As can be observed, both array antennas have a good impedance matching and isolation performance at the two operation channels. For Array-I, there are three reflection zeros at each band, exhibiting a $3^{\text {rd }}$-order filtering function. The bandwidths of the low- and high-band are $250 \mathrm{MHz}$ and $300 \mathrm{MHz}$, respectively. The isolations between them are $30 \mathrm{~dB}$ and $28 \mathrm{~dB}$. As for Array-II, four reflection zeros can be identified at each operation channel. The additional reflection zero results from the integrated filtering power dividing network. Therefore, a $4^{\text {th }}$-order filtering response is achieved. Compared with that of Array-I, the bandwidths, frequency selectivity and the isolations of the Array-II are all improved. The bandwidths of the low- and high-band operations are increased to $280 \mathrm{MHz}$ and $350 \mathrm{MHz}$. The isolations at the low- and high-band are improved by $6 \mathrm{~dB}$ and $4 \mathrm{~dB}$, respectively. It should also be noted that Array-II exhibits a sharper roll-off at both edges of the two bands, which is beneficial to the frequency selectivity.

Fig. 14 compares the simulated gain responses of the two array antennas at both operation bands. It is observed that the two antennas show a similar and flat gain curve within the passbands, but different out-of-band responses. The Array-II 


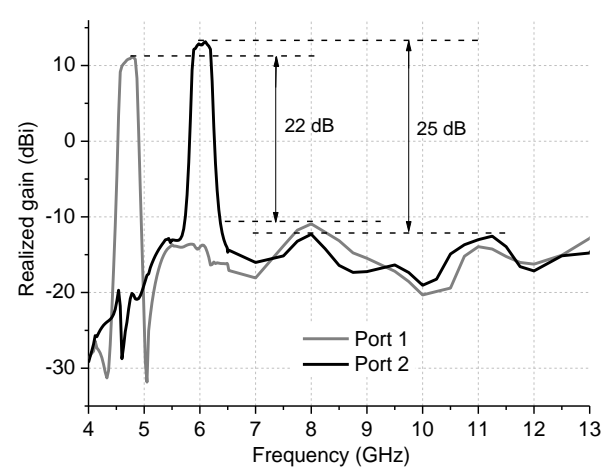

Fig. 15. Simulated antenna gains of Array-II over a broadband.

with the higher order filtering shows a sharper roll-off at the band edges and higher out-of-band rejection than that of the Array-I. For Array-II, there are two clear nulls just outside the passbands, resulting in a rapid reduction of the gain, especially for the low-band operation. At 4.4 and $5.0 \mathrm{GHz}(6 \%$ beyon the central frequency), the gain of Array-II is reduced by more than $15 \mathrm{~dB}$. Furthermore, the inter-channel rejections of the Array-II have been improved by $10 \mathrm{~dB}$ (low-band) and $4 \mathrm{~dB}$ (high-band) as compared with Array-I.

Another noticeable feature of the duplex antenna arrays is the broadband harmonics suppression. Fig. 15 shows the simulated gain curves of the Array-II over a broadband when the two ports are excited, respectively. As can be observed, the antenna has an excellent out-of-band rejection performance with the higher order harmonics significantly suppressed over a wideband up to $13 \mathrm{GHz}$. For the low-band operation (port 1 excitation), the gains at the harmonics are suppressed by more than $22 \mathrm{~dB}$. When the port 2 is excited, the gains at the harmonics are reduced by over $25 \mathrm{~dB}$.

\section{RESUlts AND Discussion}

The proposed full-duplex antenna element and the two array antennas (Array-I and II) were prototyped and measured to verify the design concepts.

\section{A. Full-duplex filtering antenna element}

Fig. 16 shows the front and back view of the full-duplex antenna element prototype. The reflection coefficients at the two ports and the isolation between them are measured and presented in Fig. 17. The measured results match very well with the simulations, showing two operation bands from 4.58 to $4.82 \mathrm{GHz}(\mathrm{FBW}=5.1 \%)$ and 5.87 to $6.2 \mathrm{GHz}(\mathrm{FBW}=$ $5.8 \%$ ) for uplink and downlink. At both bands, the antenna exhibits a $3^{\text {rd }}$-order filtering characteristics with a good frequency selectivity. Out of the bands, the antenna also shows a favorable rejection performance with the return loss close to zero. The antenna element has an excellent isolation of over $30 \mathrm{~dB}$ at both bands as indicated by $\mathrm{S}_{12}$.

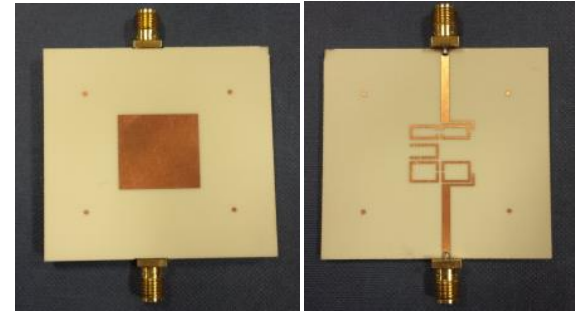

Fig. 16. Prototype of the proposed dual-port full-duplex antenna element: (a) front-view, (b) back-view.

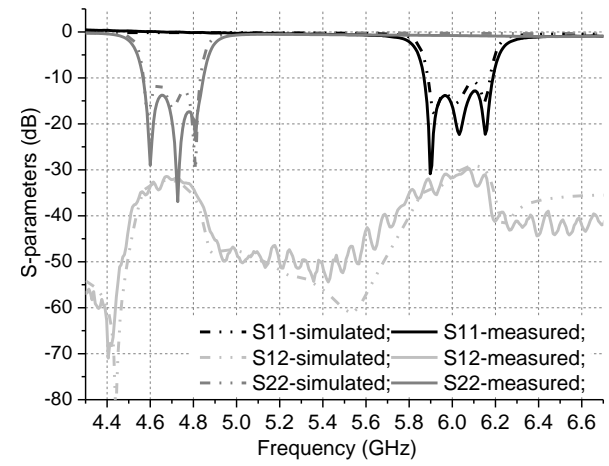

Fig. 17. Simulated and measured S-parameters of the proposed dual-port full-duplex antenna element.

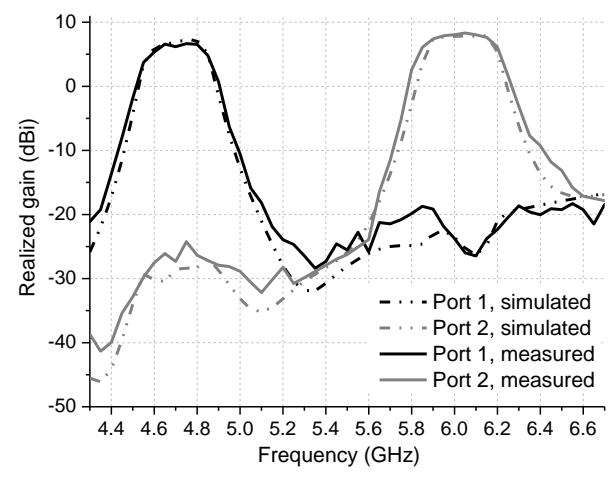

Fig. 18 shows the simulated and measured realized gains of the dual-port full-duplex antenna element when the two ports are excited, respectively. As can be observed, the measured gains agree well with the simulations. When port 1 is excited (low-band operation), the antenna has a flat gain of about 6.7 $\mathrm{dBi}$ from 4.6 to $4.8 \mathrm{GHz}$. The antenna also exhibits excellent frequency selectivity with the gain rapidly reduced to below $20 \mathrm{dBi}$ as the frequency decreases to $4.38 \mathrm{GHz}$ or increases to 


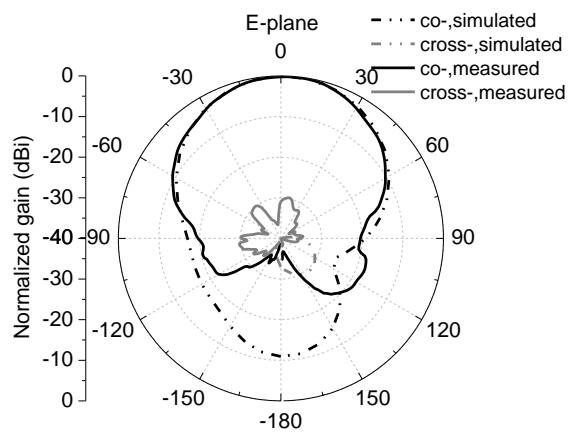

(a)

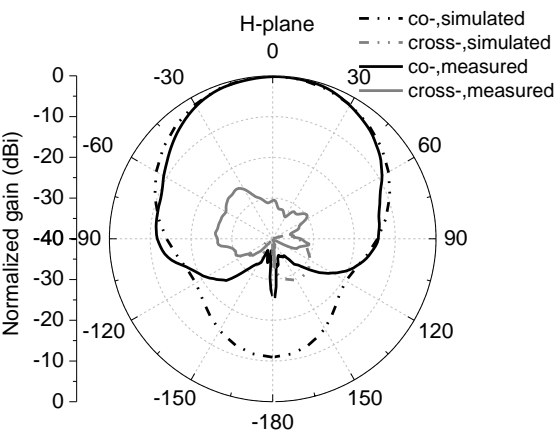

(b)

Fig. 19. Simulated and measured normalized radiation patterns of the fullduplex antenna element at $4.7 \mathrm{GHz}$ (port 1 is excited): (a) $\varphi=0^{0}$, (b) $\varphi=90^{\circ}$.

$5.08 \mathrm{GHz}$. The gain is suppressed by more than $30 \mathrm{~dB}$ at the high-band $(6 \mathrm{GHz})$. When the port 2 is excited (high-band operation), the antenna has a flat gain of about $8 \mathrm{dBi}$ from 5.88 to $6.18 \mathrm{GHz}$. The gain is significantly suppressed to below $-25 \mathrm{dBi}$ at around $6 \mathrm{GHz}$, which is $33 \mathrm{~dB}$ lower than that of in-band. The minor discrepancies between the simulations and measurements are attributed to the measurement sensitivity.

The simulated and measured normalized radiation patterns of the full-duplex antenna element at $4.7 \mathrm{GHz}$ are presented in Fig. 19. The measurements and simulations agree very well with each other, showing the maximum radiation in the broadside direction. The antenna also exhibits a very pure polarization performance with the measured cross polarization discrimination (XPD) over $30 \mathrm{~dB}$ in $\varphi=0^{0}$ (E-plane) and $\varphi=$ $90^{\circ}$ (H-plane) planes, respectively.

Fig. 20 shows the normalized simulated and measured radiation patterns of the full-duplex element at $6 \mathrm{GHz}$ when the port 2 is excited and the port 1 is terminated with $50 \Omega$ load. As can be seen, the antenna has similar radiation patterns as those in the low-band operation. The measured XPD are

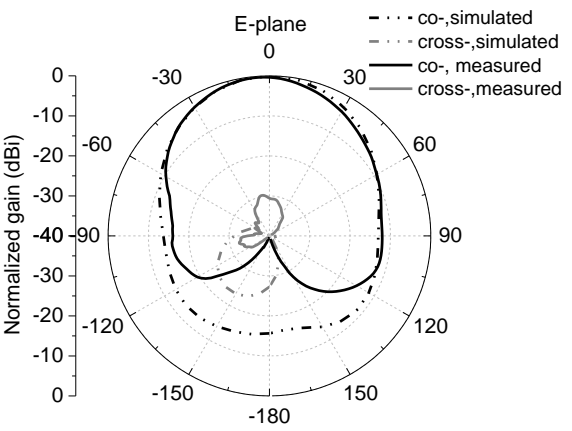

(a)

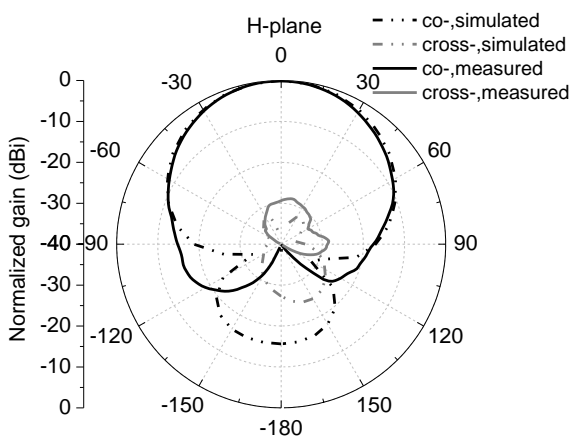

(b)

Fig. 20. Simulated and measured normalized radiation patterns of the fullduplex antenna element at $6 \mathrm{GHz}$ (port 2 is excited): (a) $\varphi=0^{\circ}$, (b) $\varphi=90^{\circ}$.

over $30 \mathrm{~dB}$ in $\varphi=0^{0}$ and $\varphi=90^{\circ}$ planes. It should be noted that the discrepancies in the backward radiation are caused by the absorber used to mount the antenna in the chamber.

\section{B. Full-duplex antenna arrays}

The two dual-port full-duplex antenna arrays (Array-I and II) were also prototyped and displayed in Fig. 21. The two arrays have the same top layer but different bottom layers. Fig. 22 shows the simulated and measured S-parameters of the Array-I. As can be seen, the simulated and measured Sparameters agree well with each other with three identifiable poles in both operation bands. For the low-band operation, the antenna has a bandwidth from 4.57 to $4.82 \mathrm{GHz}$. When port 2 is excited, the antenna works from 5.86 to $6.2 \mathrm{GHz}$. Between the two operation bands (ports), the antenna achieves a high isolation with the measured $\mathrm{S}_{21}$ lower than -30 and $-28.5 \mathrm{~dB}$ at the low- and high-band, respectively.

Fig. 23 shows the simulated and measured S-parameters of the Array-II. As expected and different from Array-I, Array-II exhibits $4^{\text {th }}$-order filtering characteristics for both operations with four reflection zeros in each band. The increased order results in wider bandwidths, higher isolation and sharper roll- 


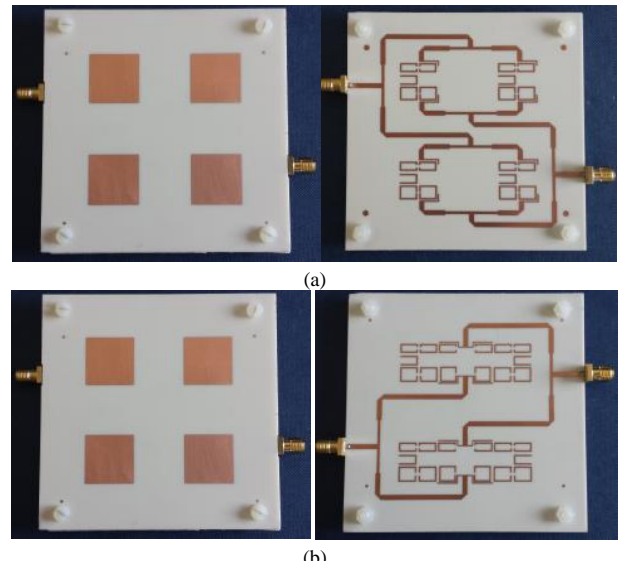

Fig. 21. The front- and back-view of the full-duplex antenna arrays: (a) Array-I, (b) Array-II.

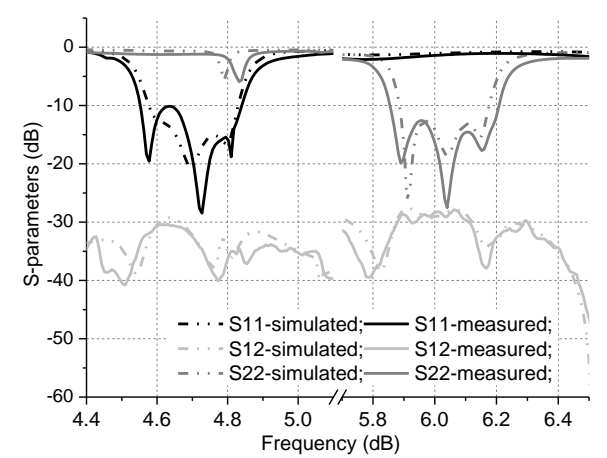

Fig. 22. Simulated and measured S-parameters of the proposed Array-I.

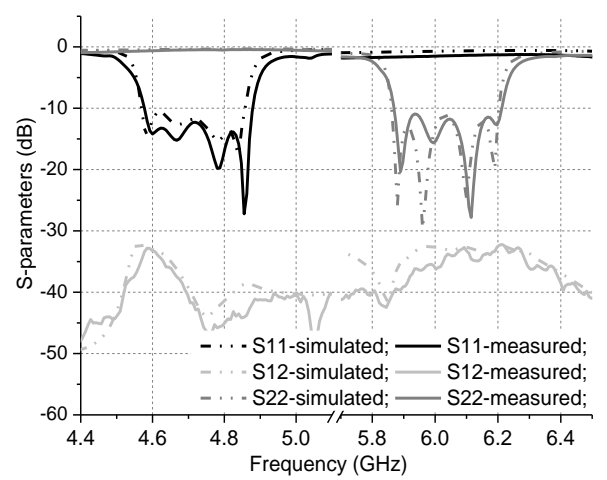

Fig. 23. Simulated and measured S-parameters of the proposed Array-II.

off at the edges of the bands. For the low-band operation, a bandwidth from 4.58 to $4.86 \mathrm{GHz}$ is achieved. The isolation in

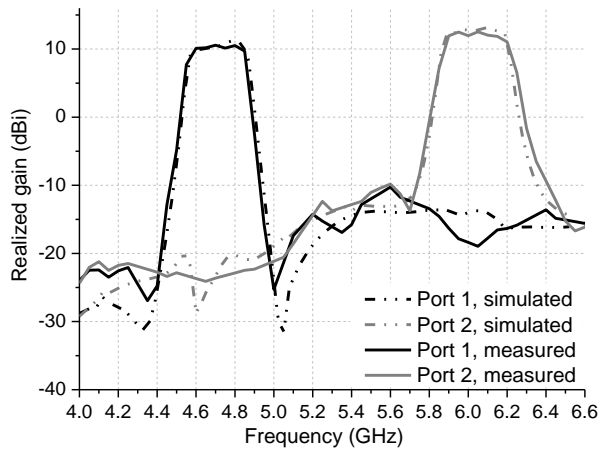

Fig. 24. The simulated and measured antenna gain of Array-II.

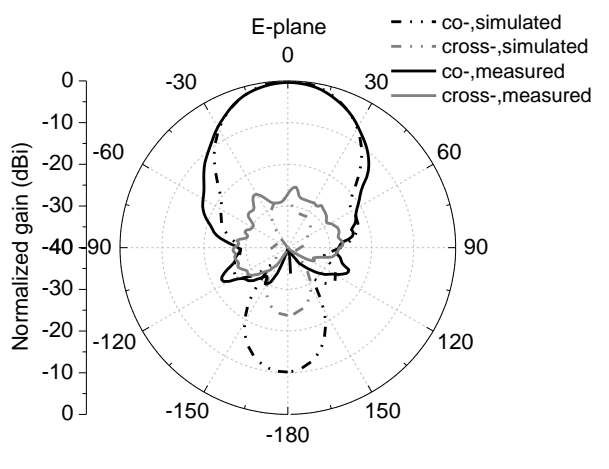

(a)

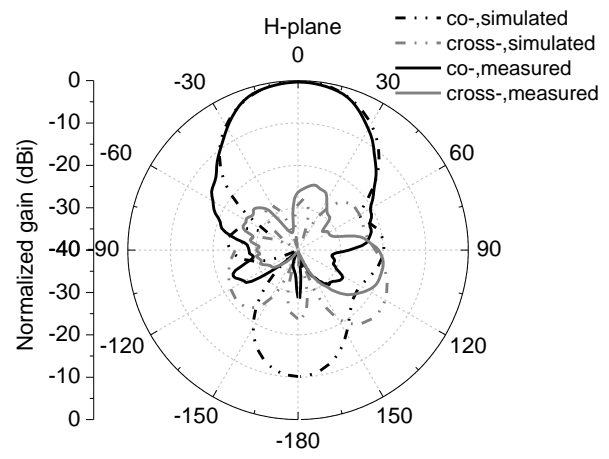

(b)

Fig. 25. Simulated and measured normalized radiation patterns of the proposed Array-II at $4.7 \mathrm{GHz}$ (port 1 is excited): (a) $\varphi=0^{\circ}$, (b) $\varphi=90^{\circ}$.

the low-band is over $33 \mathrm{~dB}$ for the measurement. When the antenna works at the high-band, a bandwidth from 5.85 to 6.22 $\mathrm{GHz}$ is obtained. The isolation in this band is higher than 32 $\mathrm{dB}$. 


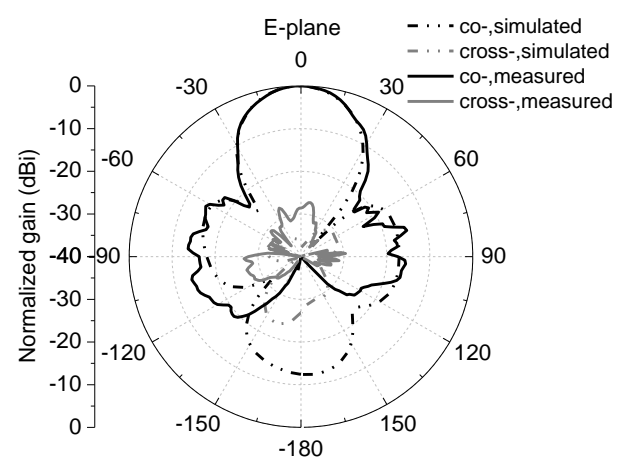

(a)

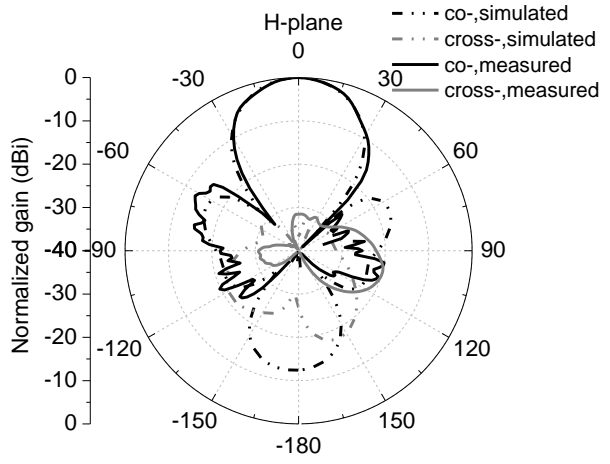

(b)

Fig. 26. Simulated and measured normalized radiation patterns of the proposed Array-II at $6.0 \mathrm{GHz}$ (port 2 is excited): (a) $\varphi=0^{\circ}$, (b) $\varphi=90^{\circ}$.

Fig. 24 shows the simulated and measured realized gains of the duplexing Array-II. (For brevity, the gains and the radiation patterns of Array-I are not presented in this paper.) The measured results agree reasonably well with the simulations. For the low-band operation, the antenna exhibits a flat gain about $10.2 \mathrm{dBi}$ from 4.6 to $4.82 \mathrm{GHz}$. At around 6 $\mathrm{GHz}$ (high-band operation), the gain is reduced to below -15 $\mathrm{dBi}$, which is more than $25 \mathrm{~dB}$ lower than that in-band. For the high-band operation, the antenna has a flat gain of $12.5 \mathrm{dBi}$ from 5.88 to $6.2 \mathrm{GHz}$. The gain is suppressed to below $-22 \mathrm{~dB}$ at around $4.7 \mathrm{GHz}$. It should also be noted that there are two nulls at both sides of each passbands, which lead to sharp rolloff at the edges of the two bands and therefore the improved frequency selectivity.

The simulated and measured normalized radiation patterns of the other full-duplex antenna array (Array-II) at the lowband are presented in Fig. 25. The measurements and simulations agree well with each other. The antenna shows a good radiation characteristics in $\varphi=0^{\circ}$ and $\varphi=90^{\circ}$ planes. The measured cross polarization discrimination (XPD) is over
TABLE-III

\begin{tabular}{ccccc}
\multicolumn{5}{c}{ COMPARISON OF THE PROPOSED FULL-DUPLEX ANTENNAS } \\
\hline \hline $\begin{array}{c}\text { Antennas } \\
\text { proposed }\end{array}$ & $\begin{array}{c}\text { Order } \\
\text { of } \\
\text { channel }\end{array}$ & $\begin{array}{c}\text { Bandwidth } \\
\text { (low-/high- } \\
\text { band) }\end{array}$ & $\begin{array}{c}\text { Isolation } \\
\text { (low-/high- } \\
\text { band) }\end{array}$ & $\begin{array}{c}\text { Inter-channel gain } \\
\text { suppression (low- } \\
\text { /high-band) }\end{array}$ \\
\hline Element & $\begin{array}{c}3^{\text {rd }} \text { - } \\
\text { oder }\end{array}$ & $5.1 \% / 5.4 \%$ & $32 \mathrm{~dB} / 30 \mathrm{~dB}$ & $30 \mathrm{~dB} / 36 \mathrm{~dB}$ \\
Array-I & $\begin{array}{c}3^{\text {rd }}- \\
\text { oder }\end{array}$ & $5.3 \% / 5.7 \%$ & $30 \mathrm{~dB} / 28 \mathrm{~dB}$ & $-/-$ \\
Array-II & $\begin{array}{c}4^{\text {th }} \\
\text { order }^{2}\end{array}$ & $6.0 \% / 6.1 \%$ & $35 \mathrm{~dB} / 33 \mathrm{~dB}$ & $26 \mathrm{~dB} / 38 \mathrm{~dB}$ \\
\hline
\end{tabular}

28 and $27 \mathrm{~dB}$ in E-plane $\left(\varphi=0^{\circ}\right)$ and H-plane $\left(\varphi=90^{\circ}\right)$, respectively.

Fig. 26 presents the normalized radiation patterns of the Array-II at $6 \mathrm{GHz}$ (high-band operation) when port 2 is excited and port 1 is terminated with a $50 \Omega$ load. As can be observed, the antenna shows the expected radiation patterns with the maximum radiation also in the broadside. However, the main beam is narrower than that of the low-band operation due to the larger electrical size of the aperture at the higher frequency. The measured XPD of the array are over $29 \mathrm{~dB}$ and $32 \mathrm{~dB}$ in $\varphi=0^{\circ}$ and $\varphi=90^{\circ}$ planes, respectively.

The results of the three dual-port full-duplex antennas are summarized in Table-III for comparison. The comparison is focused on the filtering orders of the channel, bandwidths, isolation and inter-channel gain suppression. It can be seen that the element and Array-I have similar bandwidths whereas the bandwidth of the Array-II is slightly increased due to the higher order filtering channels $\left(4^{\text {th }}\right.$-order versus $3^{\text {rd }}$-order $)$. The isolation of the Array-II at the low- and high-band are 35 and $33 \mathrm{~dB}$, which is an increase of $5 \mathrm{~dB}$ compared with the ArrayI. The results achieved have met the specifications in Table-I.

\section{CONCLUSION}

In this paper, a novel dual-port highly integrated full-duplex antenna/array with filtering characteristics were exploited to meet the operation scenarios of the vehicular communication. Such integrated RF frontend could significantly reduce the volume and cost of the Tx/Rx modules by combining the $\mathrm{Rx}$ and $\mathrm{Tx}$ antennas and removing the separate bandpass filters of the transceiver. The concept and design methods for achieving the full-duplex antenna element and arrays were detailed. The technical contributions also include the generation of the additional transmission zeros, which improve the in-band frequency selectivity and out-of-band rejection. Another advantage of this integrated filtering duplex antenna is the removal of the transmission-line based interfaces and the losses associated with them. The measured results verified the design concepts, showing the excellent performance in terms of bandwidths, isolation, radiation patterns, gains and harmonics suppression.

\section{REFERENCES}

[1] S. Biswas, R. Tatchikou, F. Dion, "Vehicle-to-vehicle wireless communication protocols for enhancing highway traffic safety," IEEE Communications Magazine, vol. 44, no. 1, pp. 74-82, Jan. 2006. 
[2] S. Karagiannis, O. Altintas, E. Ekici, G. Heijenk, B. Jarupan, K. Lin, T. Weil, "Vehicular Networking: A Survey and Tutorial on Requirements, Architectures, Challenges, Standards and Solutions" IEEE Communications Surveys \& Tutorials, vol. 13, no. 4, pp. 584616, Apr. 2011.

[3] N. Wisitpongphan, O. K. Tonguz, J. S. Parikh, P. Mudalige, F. Bai, V. Sadekar, "Broadcast storm mitigation techniques in vehicular ad hoc Sadekar, "Broadcast storm mitigation techniques in vehicular ad hoc
networks," IEEE Wireless Communications, vol. 14, no. 6, pp. 84-94, networks,"

[4] X. Lin, X. Sun, P. Ho, X. Shen, "GSIS: A Secure and PrivacyPreserving Protocol for Vehicular Communications," IEEE Trans. Veh. Technol., vol. 56, no. 6, pp. 3442-3456, Jun. 2007.

[5] J. Kenney, "Dedicated short-range communications (DSRC) standards in the United States," Proceedings IEEE, vol. 99, no. 7, pp. 1162 $1182,2011$.

[6] D. Jiang, V. Taliwal, A. Meier, W. Holfelder, R. Herrtwich, "Design of 5.9 ghz dsrc-based vehicular safety communication," IEEE Wireless Communications, vol. 13, no. 5, pp. 36-43, May, 2006.

[7] M. Torrent-Moreno, J. Mittag, P. Santi, H. Hartenstein, "Vehicle-tovehicle communication: fair transmit power control for safety-critical information," IEEE Trans. Veh. Technol., vol. 58, no. 7, pp. 3684 3703, Jul. 2009.

[8] K. Mahler, W. Keusgen, F. Tufvesson, T. Zemen, G. Caire, "Tracking of Wideband Multipath Components in a Vehicular Communication Scenario," IEEE Trans. Veh. Technol., vol. 66, no. 1, pp. 15-25, Jul. 2017.

[9] X. Ge, H. Cheng, G. Mao, Y. Yang, S. Tu, "Vehicular communications for 5G cooperative small-cell networks," IEEE Trans. Veh. Technol., vol. 65, no. 10, pp. 7882-7894, Oct. 2016

[10] W. Viriyasitavat, M. Boban, H. M. Tsai, A. Vasilakos, "Vehicular communications: survey and challenges of channel and propagation models," IEEE Veh. Technol. Magazine, vol. 10, no. 2, pp. 55-66, Feb. 2015.

[11] L. Li, D. Wen, D. Yao, "A survey of traffic control with vehicula communications," IEEE Trans. Intell. Transp. Syst., vol. 15, no. 1, pp. 425-432, Jan. 2014

[12] K. Min, S. Park, Y. Jang, T. Kim, S. Choi, "Antenna ratio for sum-rate maximization in full-duplex large-array base station with half-duplex multiantenna users, IEEE Trans. Veh. Technol, vol. 65, no. 12 , pp. 10168-10173, Dec. 2016

[13] T. Varum, J. Natos, P. Pinho and R. Abreu, "Nonuniform broadband circularly polarized antenna array for vehicular communications," circularly polarized antenna array for vehicular communications,"
IEEE Trans. Veh. Technol., vol. 65, no. 9, pp. 7219-7227, Sep. 2016.

[14] J. R. De Luis, A. Morris, Q. Gu, and F. de Flaviis, "Tunable Duplexing Antenna System for Wireless Transceivers," IEEE Trans. Antennas and Propag., vol. 60, no. 11, pp. 5484-5487, Nov. 2012.

[15] Y. Zhang, X. Y. Zhang, L. Ye, Y. Pan, "Dual-band base-station array using filtering antenna elements for mutual coupling suppression," IEEE Trans. Antennas Propag., vol. 64, no. 8, pp. 3423-3430, Aug. 2016.

[16] C. X. Mao, S. Gao, Y. Wang, Z. Wang, F. Qin, B. Sanz, Q. X. Chu, "An integrated filtering antenna array with high selectivity and harmonics suppression," IEEE Trans. Microw. Theory Techn., vol. 64 , no. 6, pp. 1798-1805, Apr. 2016.

[17] X. Y. Zhang, W. Duan, Y. M. Pan, "High-gain filtering patch antenn without extra circuit," IEEE Trans. Antennas Propag., vol. 63, no. 12, pp. 5883-5888, Dec. 2015.

[18] C. X. Mao, S. Gao, Y. Wang, F. Qin and Q. X. Chu, "Multi-Mode Resonator-Fed Dual Polarized Antenna Array with Enhance Bandwidth and Selectivity," IEEE Trans. Antennas and Propag., vol. 63, no. 12 , pp. 5492-5499, Dec. 2015

[19] P. Choeng, K. F. Chang, W. W. Choi, K. W. Tam, "A Highly Integrated Antenna-Triplexer With Simultaneous Three-Port Isolation Based on Multimode Excitation," IEEE Trans. Antennas and Propag. vol. 63, no. 1, pp. 363-386, Jan. 2015

[20] C. X. Mao, S. Gao, Y. Wang, F. Qin and Q. X. Chu, "Compact highly integrated planar duplex antenna for wireless communications," IEE Trans. Microw. Theory Techn., vol. 64, no. 7, pp. 2006-2013, Jul. 2016.

[21] J. S. Hong and M. J. Lancaster, Microwave Filger for RF/Microwave Application. New York: Wiley, 2001

[22] X. Shang, Y. Wang, W. Xia, M. J. Lancaster, "Novel Multiplexe Topologies Based on All-Resonator Structures," IEEE Trans. Microw. Theory Techn., vol. 61, no. 11, pp. 3838-3845, Nov. 2013. 